\section{Hämin-Montmorillonit und seine Bedeutung für die Festlegung der oberen Temperatur- grenze bei der Bildung des Erdöls}

\author{
Von Armin Weiss und G. Roloff
}

Anorganisch-Chemisches Institut der Universität Heidelberg (Z. Naturforschg. 19 b, 533-534 [1964] ; eingegangen am 24. März 1964)

Frühere Untersuchungen über die Einlagerung von Proteinen aus Blutserum in das Kristallinnere von Montmorilloniten zeigten, daß neben dem Albumin auch Hämoglobin aufgenommen ${ }^{1}$ und das Hämin dort besonders fest gehalten wird ${ }^{2}$. Neue Untersuchungen mit reinem Hämin haben nun ergeben, daß auch dieses von Montmorilloniten als Kation $\mathrm{C}_{34} \mathrm{H}_{32} \mathrm{~N}_{4} \mathrm{O}_{4} \mathrm{Fe}^{\oplus}$ in das Kristallinnere eingelagert und nicht nur durch Adsorption an der äußeren Oberfläche gebunden wird. Man erhält bei dieser Einlagerung einen Hämin-Montmorillonit mit $2,8 \%$ fest gebundenem Hämin, welches sich durch siedendes Dioxan nicht mehr entfernen läßt.

Röntgenographische Untersuchungen lieferten für den bei $130{ }^{\circ} \mathrm{C}$ getrockneten Hämin-Montmorillonit einen Schichtabstand von ca. 14,6 $\AA$, während der für die Versuche verwendete Ausgangsmontmorillonit (NaMontmorillonit aus Montmorillonit von Geisenheim) nach gleichartiger Trocknung einen Schichtabstand von nur $9,8 \AA$ hatte. Eine Verringerung des Schichtabstandes wird hier also durch die Hämin-Kationen gehemmt. Bei einem Schichtabstand von $14,6 \AA$ steht für die Hämin-Kationen ein Raum von maximal 5,4 $\AA$ Höhe zur Verfügung $(14,6 \AA-9,2 \AA)$. Die scheibchenformigen Häminmolekeln müssen daher parallel zu den Silicatschichten liegen.

Mit Pyridin, das selbst als Kation und als Quellungsflüssigkeit in Montmorillonite eingelagert werden kann ${ }^{3}$, läßt sich das Hämin in Freiheit setzen. Der Schichtabstand nach dem Eluieren mit Pyridin, Waschen mit Wasser und erneutem Eintausch von Natriumionen erniedrigt sich beim Trocknen bei $130{ }^{\circ} \mathrm{C}$ wieder auf 9,9 Ångström.

Nach den Angaben von $\mathrm{CRUTE}^{4}$ über die Struktur von Ni-Ätioporphyrin ist der Flächenbedarf für ein Hämin-Kation mit etwa $216 \AA^{2}$ dreimal so groß wie die Äquivalentfläche $\left(72 \AA^{2}\right)$ für ein austauschbares einwertiges Kation. Die verfügbare Austauschkapazität sollte deshalb für diese großen Ionen auf etwa $1 / 9 \mathrm{der}$ totalen Austauschkapazität $(=0,111 \cdot 81 \mathrm{mval} / 100 \mathrm{~g}=$

1 A. Weiss u. G. Koсн, Dissertation, Techn. Hochschule Darmstadt, 1960

2 A. WeIss, Dissertation, Darmstadt 1953.

${ }^{3}$ H. v. Olphen u. J. Deeds, Nature [London] 194, 176 [1962]; A. Greene-Kelley, Trans. Farad. Soc. 51, 412, 425 [1955].

4 M. B. Crute, Acta crystallogr. 12, 24 [1959].
$9 \mathrm{mval} / 100 \mathrm{~g}$ Montmorillonit von Geisenheim) absinken. Der gefundene Wert von $4,2 \mathrm{mval} / 100 \mathrm{~g}$ Montmorillonit liegt etwa in dieser Größenordnung.

Die (001)-Interferenz des Hämin-Montmorillonits ist stark verbreitert. Der Schichtabstand läßt sich daher nicht mit großer Genauigkeit bestimmen. Höhere Ordnungen der (001)-Interferenzen treten nur mit sehr geringer Intensität auf. Dies und ihre Lage deuten darauf hin, daß keine völlig gleichmäßige Einlagerung des Hämins zwischen sämtliche Silicatschichten erzielt wurde. Dafür spricht auch, daß statt ca. 9 mval nur ca. 4,2 mval Hämin pro $100 \mathrm{~g}$ Montmorillonit gebunden wurden. Diese Differenz wird verständlich, wenn man berücksichtigt, daß die Diffusion der großen Kationen in dem nur ca. $5 \AA$ dicken Schichtzwischenraum sicher behindert ist.

TreibS $^{5}$ konnte schon 1934 nachweisen, daß im Erdöl und Ölschiefer Porphyrine enthalten sind. Untersuchungen anderer Forscher ${ }^{6}$ bestätigten das Vorhandensein von Porphyrinen und -derivaten in Sedimenten und bituminösen Substanzen. Dies weist auf die organische Natur der Erdölvorstufen hin. Der Nachweis dieser empfindlichen Verbindung wurde als Beweis dafür angesehen, daß die Temperatur bei der Erdölbildung nicht über $250{ }^{\circ} \mathrm{C}$, wahrscheinlich sogar nicht über $200{ }^{\circ} \mathrm{C}$ gestiegen sein kann, denn über $200{ }^{\circ} \mathrm{C}$ beginnt bereits die Decarboxylierung der im Erdöl nachgewiesenen Porphyrincarbonsäuren.

Thermische Abbauversuche unter Luftabschluß haben nun gezeigt, daß das Porphyrinsystem im Kristallinneren der Montmorillonite selbst bei längerem Erhitzen auf $300{ }^{\circ} \mathrm{C}$ erhalten bleibt (Tab. 1). Offensichtlich wirken die großen Silicatschichten, die parallel zum Häminsystem liegen, auf dieses stabilisierend. Außer dem eingesetzten Hämin findet man in derartig erhitzten Hämin-Montmorilloniten noch andere Porphyrine, darunter Mesoporphyrin, Hämatoporphyrin und Ätioporphyrin, die auch aus natürlichen Erdölen und Ölschiefern isoliert werden konnten.

Der Chemismus der Reaktionen, welcher im Kristallinnern des Montmorillonits vom Hämin zum Meso-, Hämato- und Ätioporphyrin führt, ist noch nicht erforscht. Er wird besonders unübersichtlich, weil bei kurzzeitigem Erhitzen (15 Stdn.) auf $250^{\circ}$ und $300{ }^{\circ} \mathrm{C}$ auch noch andere Porphyrine in sehr kleinen Mengen entstehen.

Von verschiedenen Seiten wird heute angenommen, daß zwischen den glimmerartigen Schichtsilicaten und

5 A. Treibs, Liebigs Ann. Chem. 509, 103 [1934]; 510, 42 [1934] ; 517, 172 [1935]; Angew. Chem. 49, 682 [1936]; Erdöl u. Kohle 1, 137 u. 185 [1948].

6 M. Blumer, Helv. chim. Acta 33, 1627 [1950]; J. W. Moore u. H. N. Dunning, Ind. Engng. Chem. 47, 1440 [1955]. 


\begin{tabular}{|c|c|c|c|c|}
\hline $\begin{array}{c}\text { Er- } \\
\text { hitzungs- } \\
\text { temp. } \\
{\left[{ }^{\circ}\right]}\end{array}$ & $\begin{array}{c}\text { Er- } \\
\text { hitzungs- } \\
\text { dauer } \\
\text { [Stdn. }]\end{array}$ & $\begin{array}{c}\text { Montmorillonit nachgewiesen * } \\
\text { Mämin }\end{array}$ & $\begin{array}{c}\text { Mesopor- } \\
\text { phyrin }\end{array}$ & $\begin{array}{c}\text { Ätiopor- } \\
\text { phyrin }\end{array}$ \\
\hline & 15 & ++++ & - & + \\
200 & 50 & ++++ & - & + \\
& 150 & ++++ & + & + \\
& 15 & ++++ & + & + \\
250 & 50 & +++ & + & ++ \\
& 150 & +++ & + & ++ \\
& 15 & +++ & + & ++ \\
300 & 50 & +++ & + & ++ \\
& 150 & ++ & + & +++ \\
\hline
\end{tabular}

Tab. 1. Verhalten von Hämin-Montmorillonit beim Erhitzen. ++++ eingesetzte Mengen nahezu quantitativ gefunden, +++ größere Mengen, ++ geringe Mengen, + Spuren.

* Nachweis papierchromatographisch nach R. KeHL und W. Sтісн (Hoppe-Seyler's, Z. physiol. Chem. 289, 6 [1951]) mit 2.6-Dimethyl-pyridin-Wasser (1:1) als Laufmittel.

7 C. E. W Waver, Clays and Clay Minerals 8, 214 [1960]; I. D. Schuss, Dokl. Akad. Nauk SSSR 123, 353 [1958].

8 U. Hofmann u. W. Immel, in: W. Immel, Dissertation, Techn. Hochschule Darmstadt 1960. der Bildung von Erdöllagerstätten ein Zusammenhang besteht ${ }^{7,9}$. So erhält z. B. der Ölschiefer aus der Grube Messel bei Darmstadt, in dem Treibs ${ }^{3}$ Porphyrine nachgewiesen hatte, einen großen Anteil an Montmorillonit ${ }^{8}$, zwischen dessen Silicatschichten die verschiedensten organischen Verbindungen eingelagert sind. Die Extraktion von Häminderivaten aus solchen Schiefern erlaubt aber nicht zu folgern, daß bei der Entstehung des Erdöls die Temperatur nicht über $200-250{ }^{\circ} \mathrm{C}$ angestiegen sein kann. Wenn man aus diesen Vorkommen von Hämin auf die obere Temperaturgrenze während der Erdölentstehung schließen möchte, sollte sie wegen der stabilisierenden Wirkung der Silicatschichten um mindestens $100{ }^{\circ} \mathrm{C}$ nach oben verschoben werden. Damit spricht kein experimenteller Befund mehr gegen die Annahme solcher höheren Temperaturen von $200-300{ }^{\circ} \mathrm{C}$, bei denen die Schichtsilicate als Katalysatoren für die Hydrierung, Isomerisierung, Alkylierung und Crackung wirken. Man muß daher auch eine direkte Beteiligung der quellungsfähigen Schichtsilicate bei der Entstehung des Erdöls in Betracht ziehen ${ }^{9}$.

9 A. Weiss u. G. Roloff, Internat. Clay Conf. 1963, 2, im Druck.
Beiträge zur Chemie des Schwefels, $69^{1}$

\section{Über Charge-Transfer-Beziehungen zwischen Schwefel und Jod}

\author{
Von K.-H. Linke
}

Institut für Anorganische Chemie der Universität Köln (Z. Naturforschg. 19 b, $534-535$ [1964] ; eingegangen am 25. März 1964)

Einige anorganische und organische Jodide bilden mit Schwefel feste Additionsverbindungen der allgemeinen Formel $\mathrm{RJ}_{\mathrm{n}} \cdot \mathrm{nS}_{8}$. Kristallstruktur-Untersuchungen an $\mathrm{CHJ}_{3} \cdot 3 \mathrm{~S}_{8}{ }^{2}$ und $\mathrm{SbJ}_{3} \cdot 3 \mathrm{~S}_{8}{ }^{3}$ führten zu der Ansicht, daß diese Verbindungen zu den sog. Charge-TransferKomplexen ${ }^{4}$ gehören. Bei Ersatz von $\mathrm{S}_{8}$ durch $\mathrm{S}_{7} \mathrm{NH}$, $\mathrm{S}_{4} \mathrm{~N}_{4}$ oder $\mathrm{S}_{4}(\mathrm{NH})_{4}$ konnten keine entsprechenden Additionsverbindungen erhalten werden ${ }^{5}$, so daß zur Verbindungsbildung möglicherweise der gesamte $\mathrm{S}_{8}$-Ring erforderlich ist. Zur Diskussion dieser Frage wurden spektroskopische Untersuchungen durchgeführt.

Absorptionsspektren im ultravioletten und sichtbaren Bereich wurden von $\mathrm{CHJ}_{3} \cdot 3 \mathrm{~S}_{8}, \mathrm{AsJ}_{3} \cdot 3 \mathrm{~S}_{8}, \mathrm{C}_{2} \mathrm{~J}_{4} \cdot 4 \mathrm{~S}_{8}$, $\mathrm{P}_{2} \mathrm{~J}_{4} \cdot 4 \mathrm{~S}_{8}$ und von Mischungen von $\mathrm{CHJ}_{3}$ und $\mathrm{S}_{8}(1: 1$, $1: 2,1: 3)$, von $\mathrm{CHJ}_{3}$ und $\mathrm{S}_{7} \mathrm{NH}(1: 1,1: 2,1: 3)$, von $\mathrm{J}_{2}$ und $2 \mathrm{~S}_{8}$ und von $\mathrm{J}_{2}$ und $2 \mathrm{~S}_{7} \mathrm{NH}$, jeweils gelöst in Cyclohexan, aufgenommen. In einigen Fällen traten geringe Verschiebungen von Absorptionsbanden der

1 68. Mitt. F. FehÉr u. K.-H. Linke, Chem. Ber. (im Druck).

2 T. Buorvatten, Acta chem. scand. 16, 749 [1962].

3 T. Buorvatten, O. Hassel u. A. Lindheim, Acta chem. scand. 17, 689 [1963].
Komplexkomponenten zu kürzeren Wellenlängen hin auf. So wurde an Mischungen von Jodoform und Schwefel bei der Zusammensetzung $\mathrm{CHJ}_{3}+2 \mathrm{~S}_{8}$ im Vergleich mit anderen Mischungsverhältnissen eine maximale Blauverschiebung $(5 \mathrm{~m} \mu)$ festgestellt, die auf das Vorliegen eines Molekelkomplexes $\mathrm{CHJ}_{3} \cdot 2 \mathrm{~S}_{8}$ in der Lösung hinweisen könnte. Die IR-Spektren $(1-24 \mu)$ der oben angeführten Additionsverbindungen zeigten nur bei den festen Substanzen deutliche Veränderungen der Absorptionsbanden der Komplexkomponenten. Es traten Rotverschiebungen auf, die z. B. bei Banden des Jodoforms $23 \mathrm{~cm}^{-1}$ bzw. $5 \mathrm{~cm}^{-1}$ betrugen.

Bei der Lösung von $\mathrm{CHJ}_{3} \cdot 3 \mathrm{~S}_{8}$ in Schwefelkohlenstoff konnten zwei neue Absorptionsbanden bei 733 $\mathrm{cm}^{-1}$ und $762 \mathrm{~cm}^{-1}$ beobachtet werden. Ihre Intensitäten erreichen etwa zwei Stdn. nach dem Lösen der Substanz ein Maximum und nehmen dann wieder ab. Auch eine Lösung von $\mathrm{P}_{2} \mathrm{~J}_{4} \cdot 4 \mathrm{~S}_{8}$ in Schwefelkohlenstoff zeigt an derselben Stelle die beiden Absorptionen neben einer dritten Bande bei $700 \mathrm{~cm}^{-1}$, die aber wohl einem Umsetzungsprodukt von Diphosphortetrajodid mit Schwefel zuzuordnen ist. Hierbei dürfte es sich allerdings nicht um zwei für einen Molekelkomplex charakteristische Absorptionsbanden handeln. Sie werden sehr wahrscheinlich durch eine Reaktion des Schwefelkohlenstoffs hervorgerufen und weisen vermutlich auf $\mathrm{C}-\mathrm{S}$. Bindungen hin.

${ }^{4}$ Vgl. hierzu G. Briegleb, „Elektronen-Donator-AcceptorKomplexe“, Springer-Verlag, Berlin- Göttingen-Heidelberg 1961.

5 F. Fehér, D. Hirschfeld u. K.-H. Linke, Acta crystallogr. 15, 1182 [1962]. 